\title{
Giant permittivity and dynamic mobility observed for spherical polyelectrolyte brushes
}

\author{
M. L. Jiménez, ${ }^{a}$ A. V. Delgado, ${ }^{* a}$ S. Ahualli, ${ }^{a}$ M. Hoffmann, ${ }^{b}$ A. Witteman ${ }^{b}$ and M. Ballauff ${ }^{c}$ \\ Received 24th December 2010, Accepted 2nd March 2011 \\ DOI: 10.1039/c0sm01544j
}

We present a study of the electrodynamic behavior of concentrated suspensions of spherical polyelectrolyte brushes (SPBs). The dynamic mobility of the SPBs exhibits giant values. Concomitantly, the dielectric spectra of suspensions of these particles display enormous loss peaks in the $\mathrm{kHz}$ frequency range. The strong dielectric relaxation is a direct consequence of the inhomogeneity of the counterion distribution inside the polyelectrolyte layer. As can be concluded from the experimentally determined relaxation frequency, the mobility of monovalent counterions is strongly diminished in the brush region. The dynamic behavior of the SPBs at high volume fractions can be explained by the assumption that the polyelectrolyte chains deform when particles approach each other.

In the last few years there has been increasing interest in the analysis of the structure and behavior of soft particles, constituted by a hard core and a polymer shell, either charged or not. Polyelectrolyte layers are often used for stabilizing nanoparticles in high ionic strength environments ${ }^{1}$ or to provide them with a biodegradable or biocompatible coating, which in addition might load and release drugs or other therapeutic agents with biomedical applications in mind. Also, ordinary latex particles usually dangle terminating chains at the surface. ${ }^{2}$ Understanding the structure and behavior of polyelectrolyte chains in these environments is essential in designing the final device. Spherical polyelectrolyte brushes (SPBs) constitute an important example of such particles. The SPB consists of a solid core onto which a layer of polyelectrolyte chains is densely grafted. ${ }^{3}$ Fig. 1 shows the structure of the SPB in a schematic fashion. For salt-free or low-salt solutions, the osmotic pressure of the confined counterions produced by the dissociation of the polyelectrolyte charged groups leads to stretching of the chains. If the ionic strength is raised, the balance of the osmotic pressure inside and outside the brush layer provokes a shrinking of the polyelectrolyte chains. ${ }^{4}$

Information on the structure and dynamics of soft particles can be obtained by analyzing the effect of external electric fields on SPBs, using for instance electrokinetic techniques like electrophoresis. ${ }^{5} \mathrm{~A}$

aDepartment of Applied Physics, Faculty of Science, University of Granada, 18071 Granada, Spain. E-mail: adelgado@ugr.es; Fax: +34 958 243214; Tel: +34958243209

${ }^{b}$ Physikalische Chemie I, Universität Bayreuth, 95440 Bayreuth, Germany ${ }^{c}$ F-I2 Soft Matter and Functional Materials, Helmholtz-Zentrum Berlin, Glienicker Str. 100, 14109 Berlin, Germany clear example is found in ref. 3, where indirect proofs of the structural changes experienced by SPBs when the ionic composition of the medium is modified, were obtained by means of electrophoretic mobility determinations. These authors provided strong evidence of the behavior of the SPBs as rigid colloids rather than soft particles in the presence of $\mathrm{Eu}^{3+}$ counterions. This is a consequence of the suppression of electro-osmosis in the polyelectrolyte brush because of the strong binding of the multivalent counterions to the polymer chains.

In the case of monovalent counterions, although the ionic mobility can be reduced, electro-osmotic flows cannot be fully neglected as with trivalent ions, ${ }^{3}$ this fact giving a primary role to the dynamics of ions in the brush. As classical electrokinetics tell us, ${ }^{5}$ since electroosmosis and electrophoresis have the same origin, they are coupled phenomena. The velocity of the electro-osmotic flow far from the particle in a reference frame sitting on it is equal and opposite to the electrophoretic velocity of the particle in the laboratory reference frame. This means that increasing, for instance, the concentration of free counterions in the polymer brush, will lead to an enhanced electro-osmosis and, hence, a larger electrophoretic velocity.

Here we examine the dynamic mobility of the SPBs, that is, the electrophoretic mobility in alternating fields. We measured the dynamic mobility for particle concentrations up to $7 \mathrm{wt} \%$ to consider the situation of highly concentrated suspensions, which in turn are the

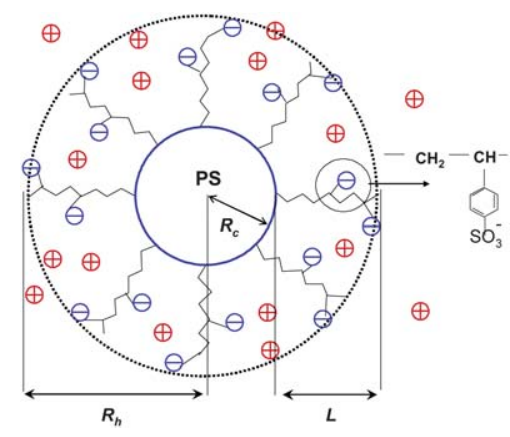

Fig. 1 Sketch of the spherical polyelectrolyte brush under consideration. The polystyrene core of radius $R_{\mathrm{c}}=50 \mathrm{~nm}$ consists of polystyrene (PS) and a thin layer of photoinitiator, onto which poly(styrene sulfonate) chains are densely grafted $\left(\sigma=0.1 \mathrm{~nm}^{-2}\right) . L(=169$ and $143 \mathrm{~nm}$ in water and $0.5 \mathrm{mM} \mathrm{NaCl}$ solution, respectively) denotes the thickness of the brush shell and $R_{\mathrm{h}}$ the hydrodynamic radius of the SPB (dashed line). 
ones most often used in practical situations. Therefore, the recently developed electrokinetic sonic amplitude (ESA) method was used, which is particularly suitable for the electrokinetic analysis of concentrated suspensions. ${ }^{6}$ The dynamic mobility can be obtained from the electroacoustic signal if the density contrast between the particles and the dispersion medium is known, as well as the volume fraction of solids. For SPBs the issue is to properly estimate their density. In this work, an average density was calculated by dividing the total mass of the PS core and the polystyrene sulfonate chains by the volume of a sphere of radius $R_{\mathrm{h}}$ (Fig. 1).

We found an intriguingly high dynamic mobility of SPBs around $-11 \times 10^{-8} \mathrm{~m}^{2} \mathrm{~V}^{-1} \mathrm{~s}^{-1}$ (Fig. 2). It is also worthwhile to consider the increasing effect of the particle concentration, which cannot be explained with current models developed for either dilute ${ }^{7-9}$ or concentrated colloidal suspensions. ${ }^{10}$ A previous evaluation of $u_{\mathrm{e}}$ by standard optical methods in the dilute regime (volume fraction $0.1 \%$ ) and a constant electric field showed much lower values $\left(-4.4 \times 10^{-8}\right.$ $\mathrm{m}^{2} \mathrm{~V}^{-1} \mathrm{~s}^{-1}$ for an ionic strength of $I=0.5 \mathrm{mM}$ ). An increase of the mobility with the particle concentration was also observed in different experimental conditions with a non-fixed surface charge ${ }^{11}$ or salt free suspensions. ${ }^{12}$ In these cases, such an increase obeys most likely changes in the particle surface charge due to competitive nonionic and anionic surfactants, ${ }^{11}$ or the balance between released and added counterions as the solids volume fraction is changed. ${ }^{12}$ For the sake of comparison, Fig. 2 also includes the dynamic mobility of suspensions of polystyrene particles (PS50) with approximately the same size as the core of the SPBs. Note that the mobility of the bare particles is much smaller, for the same frequency range, than that of the SPBs, and almost unaffected by the particle concentration. The above mentioned mobility value is on the same order of bare latex particles results (see for example ref. 13) and for particles with a hairy layer, either neutral ${ }^{14}$ or charged..$^{15}$

Furthermore, as far as we know, and except for the results reported in ref. 16, the largest values ever reported for electrophoretic

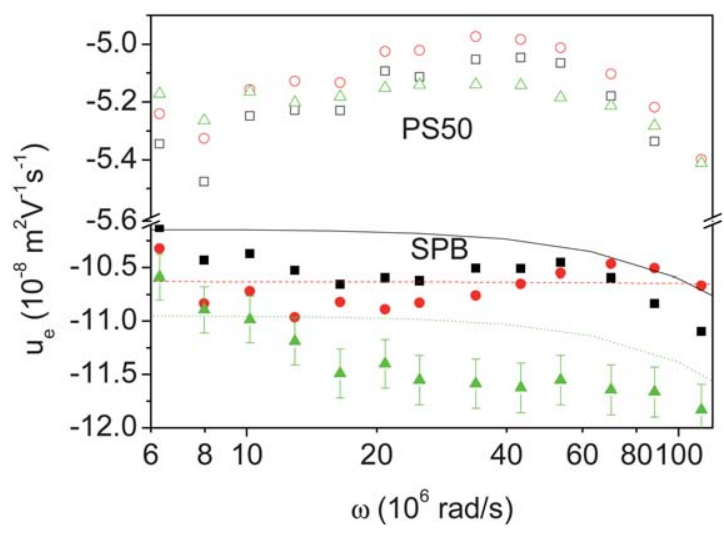

Fig. 2 Dynamic mobility of polyelectrolyte brushes (SPBs) and polystyrene spheres $50 \mathrm{~nm}$ in radius (PS50) as a function of the frequency of the applied electric field for different particle concentrations in $0.5 \mathrm{mM}$ $\mathrm{NaCl}$. Symbols denote the experimental results. Lines are the result of model predictions (see text below) with the following parameters: Particle charge: $-5 \times 10^{-16} \mathrm{C}$, polymer charge plus condensed counterions charge: $Q=-8.5 \times 10^{-14} \mathrm{C}$, dimensionless friction parameter: $\lambda R_{\mathrm{c}}=110$ at the particle surface. Squares and solid line: $5 \mathrm{wt} \%$. Circles and dashed line: $6 \mathrm{wt} \%$. Triangles and dotted line: $7 \mathrm{wt} \%$. The dispersion in these measurements is around $2-3 \%$. mobilities are around $7 \times 10^{-8} \mathrm{~m}^{2} \mathrm{~V}^{-1} \mathrm{~s}^{-1}$ in absolute value, even in the case of DNA and other polyelectrolyte molecules, much smaller than the SPBs here examined ${ }^{17}$ On the other hand, current models for hard colloidal particles tell us that there is a maximum value that cannot be surpassed whatever the charge of the particle or the salt content. ${ }^{7}$ This maximum is explained by the balance between the electric force due to the external field and that of the dipolar fields generated by the disturbed ionic atmosphere.

That the mobility of polyelectrolyte-coated particles can be larger than that of bare ones with comparable geometry was already found by Hill et al. ${ }^{9}$ in those cases in which the charge of the coating is high and so is its permeability. The latter two conditions are very likely fulfilled by our polymer brushes, but the dc mobility that we find is not that large. This means that the high dynamic mobility must be explained by other reasons, not just the existence of the charged hairy layer.

The large difference between hard particles and SPB results points to the existence of non-negligible electro-osmotic flows in the polyelectrolyte brush, produced by mobile counterions in it. This is compatible with previous results showing that although the mobility of monovalent counterions inside the brush layer can be diminished as compared to free ions, it can lead to fluid flows in the presence of applied fields., ${ }^{3,18}$

In order to obtain information about the dynamics of ions inside the brush layer, we performed another series of experiments aimed at the determination of the frequency spectrum of the electric permittivity of SPB suspensions. For the experimental evaluation, we put the sample in a thermostatted conductivity cell between two platinized platinum electrodes connected to the four terminal impedance analyzer HP4284A (Hewlett Packard, USA) with which the complex impedance is obtained. Details of the method can be found in ref. 19. The applied electric field in these measurements is around $10 \mathrm{~V} \mathrm{~m}^{-1}$, much lower than that required to form particle chains through dipole-dipole interaction. Then, the logarithmic derivative method ${ }^{20}$ was used to obtain the electric permittivity spectra. This is one of the existing techniques for reducing or eliminating the parasitic effects associated with electrode polarization at low frequency, and it has been shown to yield data quality comparable to that achieved with four-electrode cells. In Fig. 3 we have plotted both the real $\left(\varepsilon^{\prime}\right)$ and imaginary $\left(\varepsilon^{\prime \prime}\right)$ parts of the relative permittivity as a function of frequency for SPB suspensions at a concentration of $4 \mathrm{wt} \%$. The observed relaxation in Fig. 3 is unexpectedly huge and slow as compared to the permittivity spectra of rigid latex particles with a size similar to that of the core of the SPB (PS50 in Fig. 3) and to that of the whole SPB (PS168). The results on bare particles are similar to those found in literature. ${ }^{13}$ It is also enormous as compared with the decay observed for polyelectrolyte solutions, ${ }^{21}$ which never amounts to more than a few tens.

It may be recalled that the electrokinetic properties depend on the charge distribution at the interface, which typically is distorted with respect to its equilibrium conformation. When applying an ac field to a charged colloidal particle in solution, inhomogeneous fluxes are generated, which, in turn, build up an accumulation of free counterions on one side of the particle and a depletion on the other side..$^{22,23}$ This modifies the field-induced dipole at the particle/solution interface. If the frequency of the field is low enough, a gradient of neutral electrolyte concentration (the concentration polarization) is established outside the double layer, that in turn generates volume diffusion fluxes. These fluxes (mainly formed by free counterions 


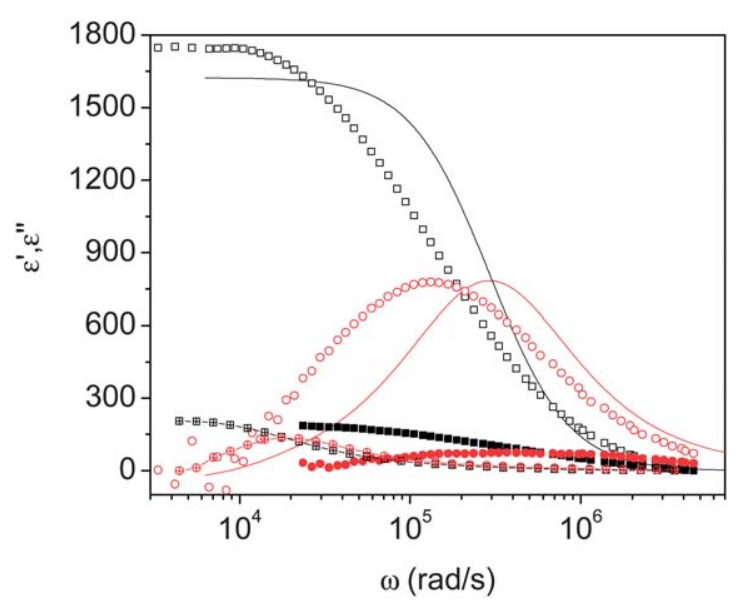

Fig. 3 Real (squares) and imaginary (circles) parts of the relative permittivity of suspensions of SPBs (open symbols), PS50 (filled symbols) and PS168 (crossed symbols) as a function of the frequency of the applied field. Particle concentration: $4 \mathrm{wt} \%$. Ionic strength $0.5 \mathrm{mM}(\mathrm{NaCl})$. Lines are the model predictions with the same parameters as in Fig. 2 and particle concentration $4 \mathrm{wt} \%$.

inside the double layer) lag behind the field and manifest as a large low-frequency electric permittivity. Above a characteristic frequency in the $\mathrm{kHz}$ range, concentration gradients cannot occur and the permittivity is greatly reduced. The phenomenon is known as $\alpha$ relaxation of the permittivity and its characteristic frequency is given by the time required for the ionic diffusion through distances of the order of the SPB size. In the brush layer, free counterions contribute to this relaxation, whereas condensed counterions fluctuate along the polyelectrolyte chains contributing to an enhanced electric dipole. ${ }^{21,24}$ These fluctuations relax also in the $\mathrm{kHz}$ range and overlap the $\alpha$ relaxation. However, the condensed counterion relaxation is far smaller than the $\alpha$-relaxation and can be neglected.

Furthermore, the amplitude of the relaxation is larger for the case of SPBs than for the polystyrene spheres (PS168) and polyelectrolyte solutions. ${ }^{21,24}$ It could be argued that this is a consequence of the possibly different values of the charges in both types of particles. However, the large difference between rigid and soft particles results can be ascribed to the presence of a volume distributed charge in the case of soft particles. In fact, for rigid particles with whatever surface charge density, there is a limiting value of the $\alpha$-relaxation amplitude given by: ${ }^{5,22}$

$$
\delta \varepsilon \sim \frac{9 \varepsilon_{\mathrm{m}}\left(\kappa R_{\mathrm{c}}\right)^{2}}{16} \phi
$$

$\delta \varepsilon$ being the amplitude of the $\alpha$-relaxation, $\varepsilon_{\mathrm{m}}$ the relative electric permittivity of the solution, $\kappa$ the inverse of the Debye length and $\phi$ the particle concentration. In this case, eqn (1) gives $\delta \varepsilon \simeq 170$, far smaller than the experimental results. Moreover, if no effect of the soft layer were expected, the characteristic $\alpha$-relaxation frequencies should be very similar in SPB and PS168 suspensions. It must be considered that the characteristic time $\tau$ of the relaxation does not depend on the particle charge. In fact, $\tau$ is the diffusion time of ions in distances of the order of the particle size $R_{\mathrm{h}}$, that is, $\tau \approx R_{h}^{2} / 2 D, D$ being the ionic diffusion coefficient. Thus the large difference in both the amplitude and relaxation time scales for the SPB and PS168 samples shown in Fig. 3 again clearly demonstrates the importance of the structure of the electromigratory fluxes inside the polyelectrolyte layer on the formation of the induced dipole.

From the dimensions of the core and the coating (Fig. 1), one can estimate that the effective volume fraction of the SPBs equals one for a critical mass fraction (CMF) of $2.8 \mathrm{wt} \%{ }^{25}$ This must be taken as an estimation based on the assumption of rigid chains, and the actual value may somewhat different, since coating can deform and change the volume embraced by every particle. In Fig. 4 we explore the relative permittivity (imaginary part) of SPB suspensions above and below the CMF. As observed, the maximum in $\varepsilon^{\prime \prime}$ increases and shifts to higher frequencies when the concentration is raised, a behavior not found in the case of rigid particles (data not shown). It must be noted that in the case of grafted polyelectrolyte with homogeneous segment distribution, the concentration polarization must be restricted to the region outside the soft layer. As a consequence, the $\alpha$-relaxation should be absent for particle concentrations above the CMF because of the close contact between neighbour brushes leaving no free volume for the concentration polarization. In fact, calculations using the model delineated in ref. 10 confirm this statement: when approaching the CMF the amplitude of the $\alpha$-relaxation decays to zero.

We must consider the necessity of taking into account the $r$-dependence of the chain density in the polyelectrolyte brush. Such an idea has been developed for dilute suspensions, ${ }^{9,26}$ but no such huge electric permittivity values were predicted. On the other hand, a cell model was used in ref. 10 to numerically solve the electrokinetic equations for a concentrated suspension of particles with a homogeneous soft layer. These equations are the classical ones modified in order to take into account the non-zero charge density inside the polymer brush (characterized by $Q$, the polymer charge plus condensed counterion charge) in the Poisson equation and an additional Stokes-like term in the Navier-Stokes equation, that accounts for the additional drag force exerted by the polyelectrolyte chains on the interstitial fluid. This last term is calculated with the Debye-

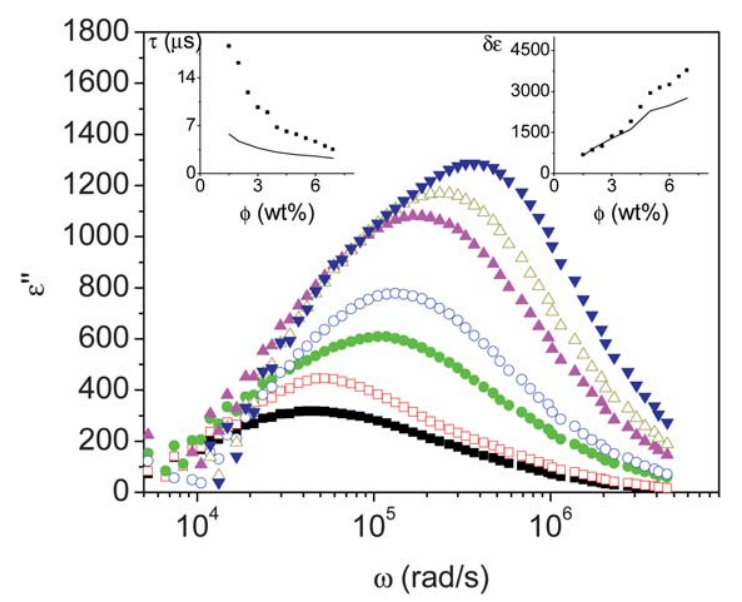

Fig. 4 Imaginary part of the relative permittivity of SPB suspensions with $0.5 \mathrm{mM} \mathrm{NaCl}$ at different particle concentrations. Full squares: $1.5 \mathrm{wt} \%$, open squares: $2 \mathrm{wt} \%$, full circles: $3 \mathrm{wt} \%$, open circles: $4 \mathrm{wt} \%$, full up triangles: $5 \mathrm{wt} \%$, open up triangles: $6 \mathrm{wt} \%$, full down triangles: $7 \mathrm{wt} \%$. Insets: characteristic time, $\tau_{\alpha}$, and amplitude, $\delta \varepsilon$, of the permittivity relaxation. Solid lines: theoretical curves obtained with the same parameters as in Fig. 2. 
Bueche model, ${ }^{8}$ that is, the chain behaves as a homogeneous group of resistance centers characterized by the parameter $\lambda=\sqrt{\gamma / \eta},{ }^{8,10}$ where $\gamma$ is the friction coefficient, and $\eta$ is the fluid viscosity. $\lambda$ is, in turn, the inverse of the Brinkman screening length $l$. The model in ref. 10 is now completed by considering that the segment density of the polyelectrolyte layer (Fig. 1) must vary as $r^{-2}$ (ref. 27). This affects both the volume charge density distribution around the particle and the Brinkman length. In the present study, the theoretical mobility and dielectric spectra of concentrated SPB suspensions have been calculated using $Q$ and $\lambda$ as the only fitting parameters.

For low to moderate volume fractions of soft particles, this model does indeed lead to a very good agreement with the experimental data on $u_{\mathrm{e}}, \delta \varepsilon$ and $\tau$ (see Fig. 2 and the insets in Fig. 4), although the characteristic frequency of the relaxation is somewhat overestimated, a fact that will be considered below. For the theoretical curves, the used parameters are, besides the geometrical characteristics, the ones detailed in the caption of Fig. 2. The free counterion charge density that we obtain is a $35 \%$ fraction of the total charge, and this corresponds to a Manning parameter $\xi_{\mathrm{M}}=3,{ }^{21}$ very near to the theoretical one $\xi_{\mathrm{M}}=\left|z_{\mathrm{p}} z_{1}\right| l_{\mathrm{B}} / b \approx 2.5\left(z_{\mathrm{p} / 1}\right.$ are the valencies of the charged group in the chain/counterions, $l_{\mathrm{B}}$ is the Bjerrum length and $b$ is the separation between two neighbor fixed charge sites in the polyelectrolyte chain). Also, we used a Brinkman screening length on the particle surface, $l=\lambda^{-1}=0.5 \mathrm{~nm}$, of the same order of the $1 \mathrm{~nm}$ theoretical value. $^{28}$

The presented modification of our theory is enough to capture the essential features of the dielectric dispersion in these systems: because the charge is not homogeneously distributed in the SPB, the concentration polarization takes place inside the polyelectrolyte brush and hence, the $\alpha$-relaxation is observable even above the CMF. In addition, the large charge of the polyelectrolyte layer, leading to intense diffusion fluxes when an external electric field is applied, explains the anomalously large relaxation amplitudes characteristic of SPB suspensions.

The second piece of information concerns the dynamics of the counterions inside the soft layer. If no effect of the soft layer on the counterion dynamics is considered, the resulting characteristic time should be smaller than the experimental value (Fig. 3 and 4). This suggests that free counterions must be slowed down by the polyelectrolyte chains, which hence act as drag centers not only for the liquid, but also for the ions. The necessary reduction in the diffusion coefficient of ions can be estimated from the relation between the characteristic frequencies of SPB and PS50 suspensions in Fig. 3. Since these frequencies are proportional to the diffusion coefficient, ${ }^{22}$ we can estimate that the latter is reduced by a factor of 1.5 in the soft layer. Similar findings for the reduced mobility of monovalent ions inside brush layers have been described in ref. 4 and 18 .

The large variation of $\tau$ with the particle concentration (Fig. 4) is associated with the interaction between particles. There is a controversy in the literature about how the brush layers interact. While some studies $^{29}$ find that brushes from different surfaces are compressed without interpenetration, others, ${ }^{30}$ based on experimental data on small angle neutron scattering, report that the corona layers interdigitize once the volume fraction exceeds some critical value. However, cryo-TEM observations by Wittemann et al. ${ }^{27}$ have shown that the brush layer tends to shrink and deform. This agrees with our permittivity data in Fig. 4. Above the CMF, the shrinking of the SPBs in contact will increase the polyelectrolyte segment density and thus the local concentration of counterions. Hence, both the characteristic dimension of the concentration polarization and the relaxation time are reduced, as observed in Fig. 4.

When the particle concentration is increased above the CMF, two effects are superimposed. On the one hand, the diffusion distance is controlled by the distance between particles and hence the diffusion time is shorter the larger the concentration is. On the other hand, compression of the polyelectrolyte layer above the CMF leads to an increased segment density. Hence, the counterion mobility is reduced, leading to a subsequent increase of the characteristic time. Below the CMF only the first effect is present, but above the CMF the second effect partially counterbalances the first one. This could be the reason for the change in tendency of $\tau$ when the volume fraction increases above $4.5 \%$ approximately (inset in Fig. 4).

The dynamic mobilities in Fig. 2 can now be explained with the same picture. Upon compression and shrinking of the brush layers above the $\mathrm{CMF}$, the counterion concentration in the vicinity of the particles will be increased. Therefore electro-osmotic flows will develop in a highly charged region. As a consequence, liquid transport will be enhanced, and so will the mobility of the particles, when the volume fraction is raised.

In conclusion, information on the distribution and the dynamics of counterions inside a grafted polyelectrolyte brush layer can be obtained by analyzing data on the dynamic mobility and the lowfrequency dielectric spectrum of concentrated SPB solutions. We have observed that the behavior of spherical polyelectrolyte brushes largely deviates, both quantitatively and qualitatively, from that of hard spheres and polyelectrolyte solutions. SPB suspensions exhibit unusually large values of both the dynamic mobility and the dielectric permittivity, which increase with particle concentration. Thus, highly concentrated suspensions of SPBs cannot be reduced to equivalent hard spheres with a well defined slip plane, and their behavior cannot be extrapolated from that of dilute systems. Also, we have shown that the experimental results are a direct demonstration of the slowing down effect of the polyelectrolyte chains on the ionic diffusivities, and of the important role of the non-homogeneous polyelectrolyte charge density inside this layer. The results presented do not contradict the hypothesis based on cryo-TEM observations that the brush layers shrink when the particles approach each other at high volume fractions.

This work was partially supported by Junta de Andalucía, Spain (Project 2008-FQM3993).

\section{References}

1 P. Pincus, Macromolecules, 1991, 24, 2912-2919.

2 J. E. Seebergh and J. C. Berg, Colloids Surf., A, 1995, 100, 139-153.

3 M. Hoffmann, A. Jusufi, C. Schneider and M. Ballauff, J. Colloid Interface Sci., 2009, 338, 566-572.

4 Y. Mei, K. Lauterbach, M. Hoffmann, O. V. Borisov, M. Ballauff and A. Jusufi, Phys. Rev. Lett., 2006, 97, 158301.

5 J. Lyklema, Fundamentals of Interface and Colloid Science, vol. II, Academic Press, London, 1995.

6 R. J. Hunter and R. W. O'Brien, in: Encyclopedia of Colloid and Surface Science, ed. A. Hubbard, Dekker, New York, 2002.

7 R. W. O'Brien and L. R. White, J. Chem. Soc., Faraday Trans. 2, 1978, 74, 1607-1626.

8 H. Ohshima, Adv. Colloid Interface Sci., 1995, 62, 189-235.

9 R. J. Hill, D. A. Saville and W. B. Russel, J. Colloid Interface Sci., 2003, 258, 56-74

10 S. Ahualli, M. L. Jiménez, F. Carrique and A. V. Delgado, Langmuir, 2009, 25, 1986-1997.

11 T. Bellini, V. Degiorgio, F. Mantegazza, F. J. Marsan and C. Scarnecchia, J. Chem. Phys., 1995, 103, 8228-8237. 
12 M. Evers, N. Garbow, D. Hessinger and T. Palberg, Phys. Rev. E. Stat. Phys., Plasmas, Fluids, Relat. Interdiscip. Top., 1998, 57, 6774 6784.

13 A. D. Hollingsworth and D. A. Saville, J. Colloid Interface Sci., 2004, 272, 235-245.

14 D. A. Saville, J. Colloid Interface Sci., 2000, 222, 137-145.

15 L. A. Rosen and D. A. Saville, J. Colloid Interface Sci., 1992, 149, $542-552$.

16 I. Semenov, P. Papadopoulos, G. Stober and F. Kremer, J. Phys.: Condens. Matter, 2010, 22, 494109.

17 D. A. Hoagland, E. Arvanitidou and C. Welch, Macromolecules, 1999, 32, 6180-6190.

18 R. Zimmermann, W. Norde, M. A. Cohen Stuart and C. Werner, Langmuir, 2005, 21, 5108-5114.

19 M. C. Tirado, F. J. Arroyo, A. V. Delgado and C. Grosse, J. Colloid Interface Sci., 2000, 221, 141-146.

20 M. L. Jiménez, F. J. Arroyo, J. van Turnhout and A. V. Delgado, J. Colloid Interface Sci., 2002, 249, 327-335.
21 F. Bordi, C. Cametti and R. H. Colby, J. Phys.: Condens. Matter, 2004, 16, R 1423-1463.

22 S. S. Dukhin and V. N. Shilov, Dielectric Phenomena and the Double Layer in Disperse Systems and Polyelectrolytes, Wiley, New York, 1974.

23 F. Carrique, F. J. Arroyo, M. L. Jiménez and A. V. Delgado, J. Chem. Phys., 2003, 118, 1945-1956.

24 F. van der Touw and M. Mandel, Biophys. Chem., 1974, 2, 218-230.

25 A. Marra, E. Peuvrel-Disdier, A. Wittemann, X. Guo and M. Ballauff, Colloid Polym. Sci., 2003, 281, 491-496.

26 J. F. L. Duval and H. Ohshima, Langmuir, 2006, 22, 3533-3546.

27 A. Wittemann, M. Drechsler, Y. Talmon and M. Ballauff, J. Am. Chem. Soc., 2005, 127, 9688-9689.

28 R. J. Hill and D. A. Saville, Colloids Surf., A, 2005, 267, 31-49.

29 N. A. Kumar and C. Seidel, Phys. Rev. E: Stat., Nonlinear, Soft Matter Phys., 2007, 76, 020801

30 A. V. Korobko, W. Jesse, S. U. Egelhaaf, A. Lapp and J. R. C. van der Maarel, Phys. Rev. Lett., 2004, 93, 177801. 